\title{
C-H FUNCTIONALIZATION IN NON-AROMATIC AZAHETEROCYCLES. AN EFFECTIVE APPROACH IN THE DESIGN OF PROMISING MOLECULES FOR MEDICINAL CHEMISTRY
}

\section{M.V. Varaksin ${ }^{1,2}$, L.A. Smyshliaeva ${ }^{1,2}$, T.D. Moseev', A.A. Akulov', V.N. Charushin ${ }^{1,2}$, O.N. Chupakhin ${ }^{1,2}$}

\author{
${ }^{1}$ Ural Federal University, 620002, Russia, Ekaterinburg, Mira str., 19. \\ ${ }^{2}$ Institute of Organic synthesis, UB RAS, 620990, Russia, Ekaterinburg, \\ S. Kovalevskaya str., 22.
}

DOI: 10.19163/MedChemRussia2021-2021-16

E-mail:m.v.varaksin@urfu.ru

$\mathrm{C}(\mathrm{sp} 2)-\mathrm{H}$ Functionalization has proven as a convenient PASE (pot, atom, step economy) strategy in medicinal chemistry to design target organic molecules of various architectures. Particularly, these transformations can be applied to modify non-aromatic azaheterocyclic systems by means of creating novel C-C and C-N bonds following metalfree nucleophilic substitution of hydrogen SNH (Scheme 1), transition metal-mediated cross-dehydrogenative coupling (CDC) reactions (Scheme 2), as well as radical-mediated cross-dehydrogenative coupling (CDC) reactions (Scheme 3) [1-4].

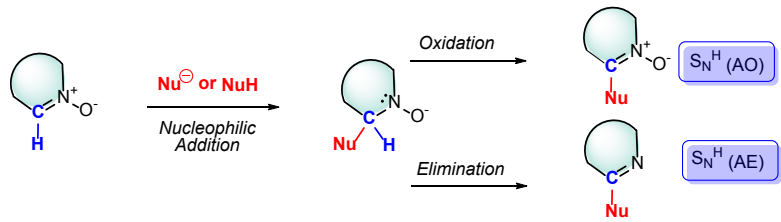

Scheme 1. Nucleophilic substitution of hydrogen reactions according to "Addition-Oxidation» $S N H(A O)$ and «Addition-Elimination» SNH(AE) protocols

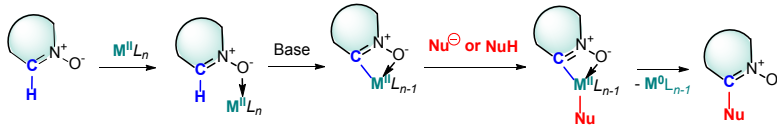

Scheme 2. Transition metal-mediated cross-dehydrogenative coupling (CDC) $M=$ Transition metal $(\mathrm{Pd}, \mathrm{Ni}, \mathrm{Cu}$, etc. $)$ reactions

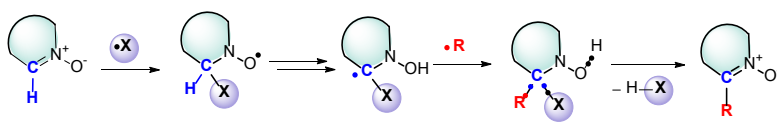

Scheme 3. Metal-free radical-mediated cross-dehydrogenative coupling $(C D C)$ reactions

The research was supported by the Russian Science Foundation (Project No. 20-43-01004).

\section{References}

[1] A.A. Akulov, M.V. Varaksin, P. Mampuys, V.N. Charushin, O.N. Chupakhin, B.U.W. Maes, Org. Biomol. Chem. 2021, 19, 297-312.

[2] A.A. Akulov, M.V. Varaksin, A.N. Tsmokalyuk, V.N. Charushin, and O.N. Chupakhin, Green Chem. 2021, 23, 2049-2057.

[3] T.D. Moseev, M.V. Varaksin, D.A. Gorlov, V.N. Charushin, O.N. Chupakhin, J. Org. Chem. 2020, 85, 11124-11133.

[4] L.A. Smyshliaeva, M.V.Varaksin, P.A. Slepukhin, O.N. Chupakhin, V.N. Charushin, Beilstein J. Org. Chem. 2018, 14, 2618-2626. 